\title{
Rehearsal in immediate serial recall
}

\author{
LYDIA TAN \\ City University, London, England \\ AND \\ GEOFF WARD \\ University of Essex, Colchester, England
}

\begin{abstract}
We report for the first time overt rehearsal data in immediate serial recall (ISR) undertaken at three presentation rates $(1,2.5$, and $5 \mathrm{sec} /$ word $)$. Two groups of participants saw lists of six words for ISR and were required either to engage in overt rehearsal or to remain silent after reading aloud the word list during its presentation. Typical ISR serial position effects were obtained for both groups, and recall increased with slower rates. When participants rehearsed, they tended to do so in a cumulative forward order up to Serial Position 4, after which the amount of rehearsal decreased substantially. There were similarities between rehearsal and recall data: Both broke down toward the end of longer sequences, and there were strong positive correlations between the maximum sequence of participants' rehearsals and their ISR performance. We interpret these data as suggesting that similar mechanisms underpin both rehearsal and recall in ISR.
\end{abstract}

In the immediate serial recall (ISR) task, participants are presented with sequences of to-be-remembered items, and, immediately after the last item is presented, try to recall as many of the items as possible in order. Typically, performance on ISR is characterized by extended primacy effects and small recency effects.

It is widely acknowledged that participants may rehearse during ISR, especially if the presentation rate is slow. For example, the working memory model assumes that rehearsal, via an articulatory control process, refreshes memory traces that would otherwise undergo rapid decay in the phonological store (Baddeley, 1986). The opposing processes of decay and rehearsal have been used widely to explain a number of phenomena such as memory-span performance, the phonological similarity effect, and the word-length effect (e.g., Baddeley, Lewis, \& Vallar, 1984; Baddeley, Thomson, \& Buchanan, 1975).

Experimenters have examined the role of rehearsal by restricting the amount of rehearsal and requiring participants to engage in articulatory suppression (i.e., continuously repeating a word such as the aloud) during list presentation. The key finding, that the phonological similarity and word length effects are eliminated under articulatory suppression with visually presented items, has been critical in specifying the detailed architecture of the working memory model (e.g., Baddeley \& Lewis, 1984; Baddeley et al., 1984).

Many formal computational models similarly assume that rehearsal occurs during ISR (see, e.g., Burgess \& Hitch, 1999; Henson, 1998; Page \& Norris, 1998). Burgess and Hitch's phonological loop model implements re- hearsal in the same way as it does serial recall, such that the two processes are equivalent. The primacy model of Page and Norris similarly models rehearsal as a cumulative process and considers rehearsals of list items simply to be more recent re-presentations of these items. They assume that participants rehearse in a cumulative forward order until such a point when rehearsal cannot be completed before the next item is presented. There is empirical evidence that a cumulative forward-order rehearsal strategy can enhance the primacy effect. Palmer and Ornstein (1971) required participants to rehearse items covertly, either cumulatively or in most recent pairs. They found that performance on a subsequent serial probed recall task was strongly affected by participants' rehearsal strategy, with much larger primacy effects being obtained in the cumulative rehearsal conditions.

Despite the widespread assumption that rehearsal plays an important role in ISR, it is perhaps surprising that no evidence exists as to which words (if any) participants rehearse. In the present experiment, we therefore sought to investigate directly the patterns of rehearsal in ISR. We used the overt rehearsal methodology, in which participants rehearse aloud during list presentation (Rundus, 1971; Tan \& Ward, 2000). Clearly, knowledge of these patterns would enable us to examine directly the assumptions inherent in models of ISR. For example, one could determine whether Page and Norris (1998) are correct in assuming that participants rehearse in a cumulative forward order until they can no longer rehearse the sequence before the next item is presented. In addition, a detailed understanding of rehearsal would allow us to examine

L.Tan, lydia.tan.1@city.ac.uk; G.Ward, gdward@essex.ac.uk 
any similarities between the mechanisms underpinning rehearsal in ISR and those underpinning recall (Burgess \& Hitch, 1999).

The overt rehearsal method necessitated presentation of the words at a slower rate than is typically used in ISR tasks. To vary the amount of rehearsal that occurred across conditions, we used three presentation rates (fast, medium, and slow). The effect of presentation rate has been shown to be ambiguous. In some cases, a slower presentation rate has led to poorer recall (e.g., Conrad \& Hille, 1958); in other studies, slower presentation rate has led to superior recall (e.g., Mackworth, 1962). These contradictory findings may perhaps have been due to the two counteracting effects of rehearsal and retention interval: Slower presentation rates are associated with increases in retention interval, but typically also lead to increased rehearsal (which should improve recall). In addition, a silent control group was used in order to determine whether the requirement to rehearse aloud affected recall.

A final advantage of overt rehearsal was that we could examine directly the effect of rehearsal on recall for each presentation rate and serial position. If rehearsal improves recall by decreasing the functional retention interval, we should see positive benefits of rehearsal to the extent that forward-cumulative rehearsal has been completed successfully throughout the list.

\section{METHOD}

\section{Participants}

Forty students from the University of Essex participated in this experiment.

\footnotetext{
Materials

The materials, selected from the Toronto Word Pool (Friendly, Franklin, Hoffman, \& Rubin, 1982), consisted of 290 disyllabic nouns, four to six letters long, with frequencies of occurrence of $75-1,199$ per million according to the Kučera and Francis (1967) norms. From this pool, 48 six-word experimental lists were selected randomly for each participant. Similarly, 3 six-word practice lists were generated. No participant received the same word twice during the experiment. The materials were presented using the SuperCard application on an Apple Macintosh computer.

Design

A mixed design was used. Type of rehearsal (overt vs. silent) was a between-subjects factor. There were two within-subjects factors: presentation rate ( $1 \mathrm{sec} /$ word [ $0.25 \mathrm{sec}$ on, $0.75 \mathrm{sec}$ off], $2.5 \mathrm{sec} /$ word [ $1.75 \mathrm{sec}$ on, $0.75 \mathrm{sec}$ off], and $5 \mathrm{sec} /$ word [ $4.25 \mathrm{sec}$ on, $0.75 \mathrm{sec}$ off ]) and serial position (1-6).

Procedure

Participants, assigned randomly to two groups of 20, were tested individually. Following the instructions, each participant saw a total of 3 practice lists followed by 48 experimental lists of six words for immediate verbal serial recall. Participants in the overt rehearsal conditions were asked to rehearse aloud any words from the list that they were thinking of, whereas participants in the silent condition were told to remain silent after reading aloud each word. Each list began with a warning tone, followed after $3 \mathrm{sec}$ by the presentation of a list. The words were presented visually one at a time in the center of the computer screen, and participants were required to say each word aloud as it appeared. A series of three beeps at the end of each list signalled the beginning of a 15 -sec recall period. Par-
}

ticipants were instructed to recall in forward serial order, and to say "blank" if they could not remember an item. At the end of the recall period, a tone sounded and participants were prompted to continue with the next list by clicking the mouse. The presentation rates were blocked, with 16 lists per presentation rate. The blocks were ordered randomly, and this ordering was used in the three practice trials.

\section{RESULTS}

\section{Recall}

Serial position. The proportion of items recalled at each serial position for each condition is shown in Figure 1. We used the standard method of ISR scoring; each response was considered to be correct if it was output in the same absolute serial position as it was input (with each "blank" response counting as an output serial position). A 2 (rehearsal type: overt or silent) $\times 3$ (presentation rate: fast, medium, or slow) $\times 6$ (serial position: $1-6$ ) mixed ANOVA was performed. This revealed a significant main effect of presentation rate $\left[F(2,76)=32.22, M S_{\mathrm{e}}=1.76\right.$, $p<.0001]$ and serial position $\left[F(5,190)=109.05, M S_{\mathrm{e}}=\right.$ $3.22, p<.0001]$, but not of rehearsal type $[F(1,138)=$ $2.88, p>.05]$.

The rehearsal type $\times$ serial position interaction was significant $\left[F(5,190)=3.23, M S_{\mathrm{e}}=0.095, p<.01\right]$, as was the presentation rate $\times$ serial position interaction $\left[F(10,380)=3.36, M S_{\mathrm{e}}=0.047, p<.001\right]$. However, the two-way rehearsal type $\times$ presentation rate interaction was not significant $(F<1)$, nor was the three-way rehearsal type $\times$ presentation rate $\times$ serial position interaction $[F(10,380)=1.21, p>.05]$.

Despite these two-way interactions, roughly similar serial position curves are found across presentation rate and rehearsal conditions. Figure 1 shows comparable levels of performance in the silent and overt rehearsal conditions, significant and extended primacy effects at all three presentation rates, and a one-item recency effect under fast and medium rates. The main differences are a slightly larger difference between Serial Positions 3 and 4 in the overt rehearsal conditions and no significant recency at the slow presentation rate. The general lack of recency may at first seem surprising, given that each item was spoken by the participant upon presentation. However, any expected modality effect may have been abolished by the participants' spoken rehearsal and recall, which may have acted as a stimulus suffix.

\section{Distribution of Rehearsal Strategies}

We analyzed the patterns of rehearsal obtained from each of the three presentation rates of the overt rehearsal conditions. We borrowed the term rehearsal set (RS) from the overt rehearsal literature in free recall (e.g., Rundus, 1971; Tan \& Ward, 2000) to refer to the set of words rehearsed after the presentation of each stimulus item. For example, the set of words in RS 4 at the slow presentation rate refers to the words rehearsed in the $5 \mathrm{sec}$ immediately after the onset of the fourth word.

We defined four rehearsal strategies: Fixed refers to the strategy of rehearsing only the most recently presented item, full cumulative refers to the strategy of rehearsing 

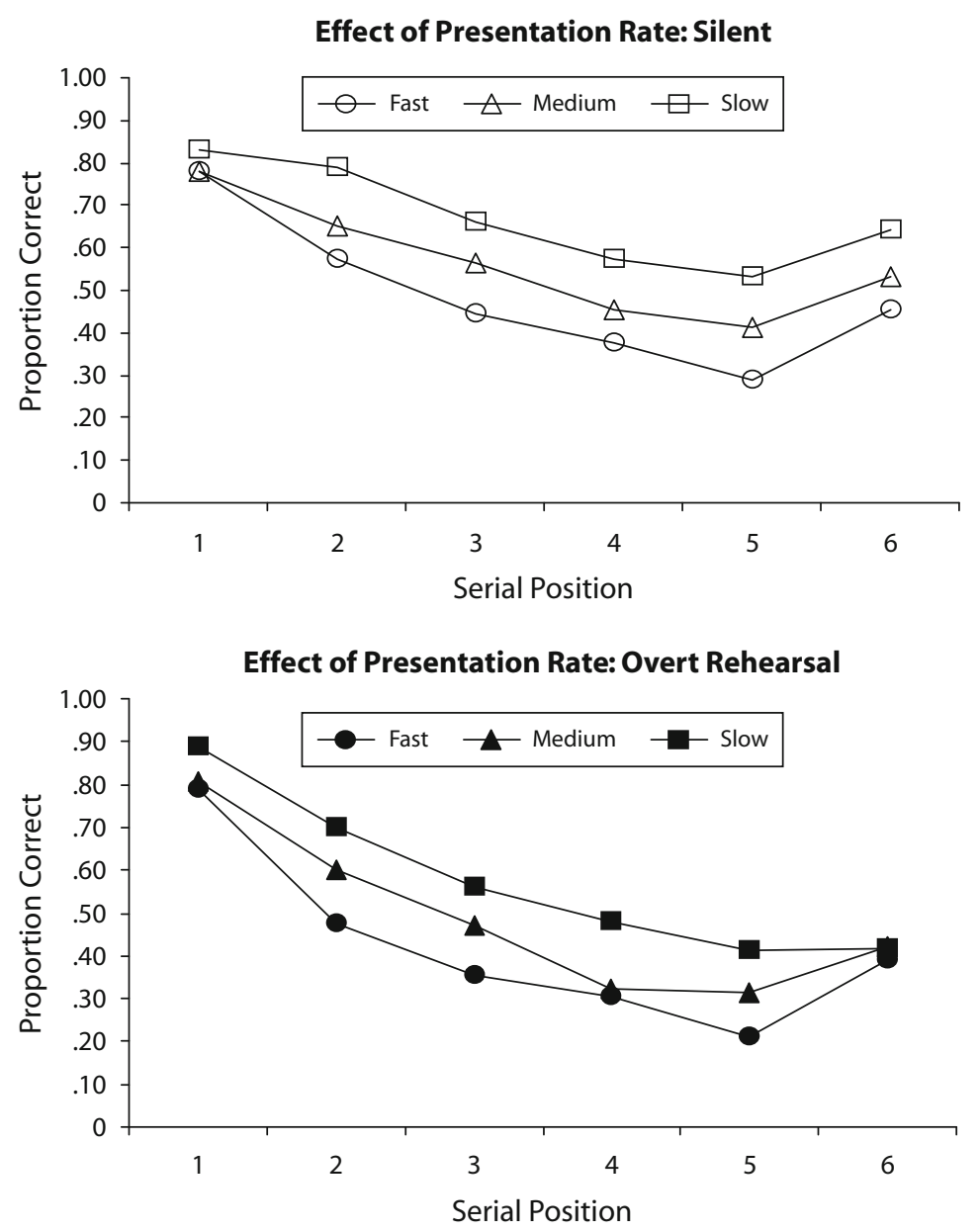

Figure 1. The serial position curves for immediate serial recall of words presented at fast, medium, and slow presentation rates in the silent condition (top panel) and overt rehearsal condition (bottom panel).

in the correct order all of the items presented to date, and partial cumulative refers to rehearsing in order only some items; any other pattern is categorized as other.

To understand how these definitions were applied, suppose a participant was presented with the sequence "A B C D E F," where the letters refer to a six-word sequence. If we take as our example an analysis of RS 4 (the sequence of rehearsals immediately following presentation of word D), a participant was deemed to have used a fixed rehearsal strategy if he or she rehearsed " $D$ " or " $D \mathrm{D}$ " or indeed repeated solely " $D$ " any number of times. A full cumulative strategy was deemed to have been used if somewhere in RS 4 participants rehearsed the string "A B C D." That is, rehearsals "D D A B C D," "A B C D A B C D," or "A B C D A B" would also be deemed full cumulative, whereas rehearsing "A B D C" or "A B C C D" would not. Similarly, a partial cumulative rehearsal strategy was deemed to have been used if somewhere in RS 4 participants rehearsed "A," "A B," or "A B C," such that rehearsing "D A B C," "A B D D," would be considered partial cumulative, but "D B C" would not.

Figure 2 shows the distribution of strategies used at each RS for each presentation rate. There are four main points to make. First, at fast rates, there was little time to rehearse, and essentially all of the rehearsals are of the most recently presented item (fixed rehearsal). Second, at medium and slow rates, there was substantial cumulative rehearsal early in the list. Over RSs $2-4$, participants adopted a full cumulative strategy approximately $45 \%$ of the time at the medium rate and approximately $70 \%$ of the time at the slow rate, but there were still significant amounts of fixed rehearsal (over $40 \%$ for the medium rate, and around $20 \%$ for the slow rate). Third, toward the end of the list at RS 5 and RS 6, cumulative rehearsal decreased and fixed rehearsal increased. Finally, there was little evidence that participants used other strategies, save toward the end of the list at the slow rate. These other responses are hard to generalize, but many contained forward-ordered sequences such as "D E" or "D E F."

\section{Rehearsal and Recall}

The relationship between rehearsal and recall was examined in three analyses. First, for each trial, a score was calculated that referred to the maximum length of sequence that was rehearsed. A score of 4 on this measure refers to a trial in which the participant rehearsed the first four words 


\section{Fast Presentation Rate: Proportion of Rehearsal Strategies Used in Each Rehearsal Set}

$\square$ Fixed

Full cumulative $\square$ Partial cumulative $\mathbf{N}$ Other

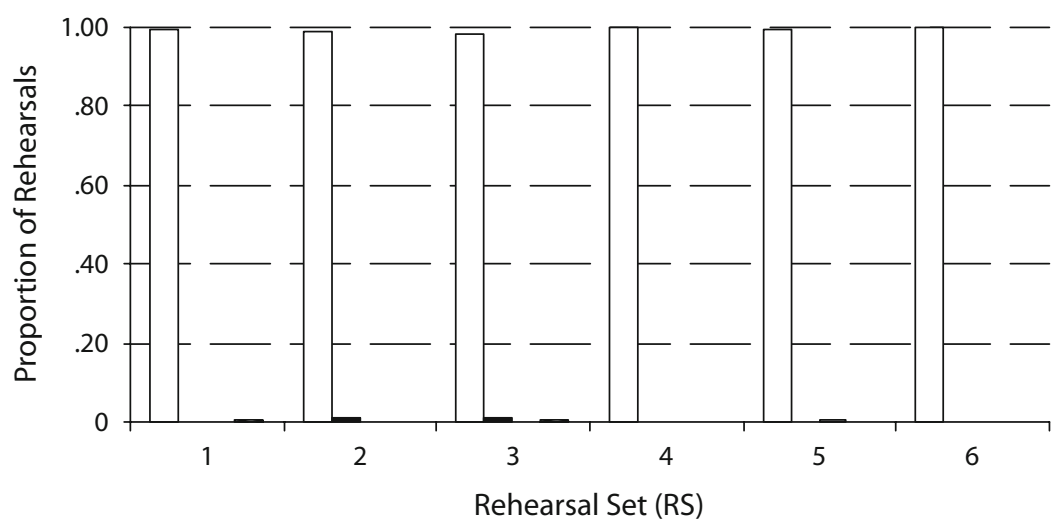

Medium Presentation Rate: Proportion of Rehearsal Strategies Used in Each Rehearsal Set

$\square$ Fixed Full cumulative $\square$ Partial cumulative $\mathbf{\nabla}$ Other

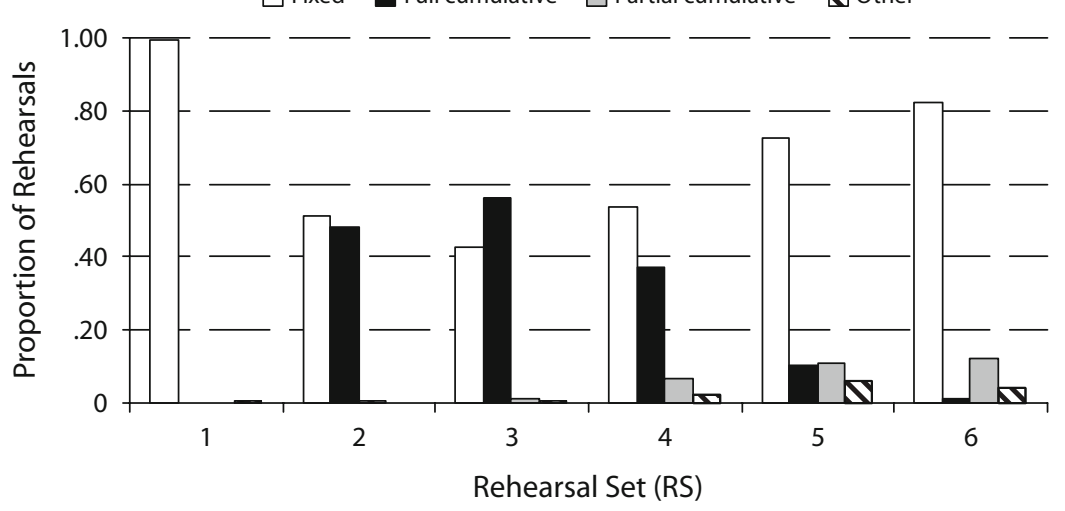

Slow Presentation Rate: Proportion of Rehearsal Strategies Used in Each Rehearsal Set

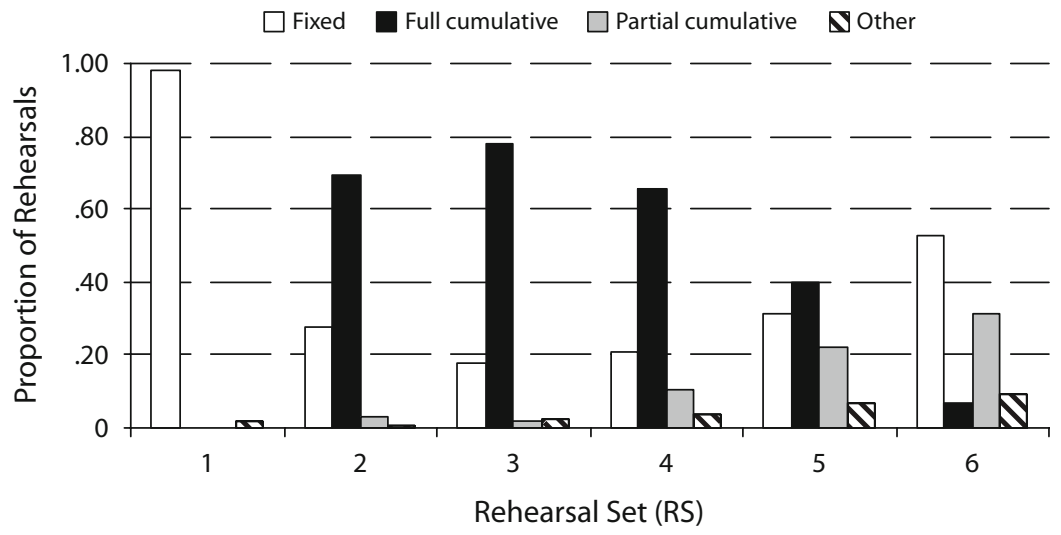

Figure 2. Data from the overt rehearsal conditions. The distributions of rehearsal strategies used during each rehearsal set (RS) are plotted separately for the fast (top), medium (middle), and slow (bottom) presentation rates. Rehearsal set refers to the set of words rehearsed immediately after the presentation of each word, such that RS 4 refers to the set of words rehearsed immediately after the presentation of the fourth word. The rehearsal strategies are described fully in the main text. 
(but neither the first five nor all six words) in the list in the correct sequence at some point during the trial; a score of 1 refers to a trial in which the participant used a fixed rehearsal strategy throughout. Figure 3 shows the relationship between this measure and ISR performance. There was not enough rehearsal to merit analysis at the fast rate, so the upper panel refers to data from the medium rate and the lower panel refers to data from the slow rate. As can be seen from the data at both rates, as the maximum sequence rehearsed increases, so does ISR performance.

Second, Figure 4 shows the ISR data across all three rates in which participants used a fixed rehearsal strategy throughout the trial. Contrary to the overall advantage of slower presentation rates on performance, participants performed better at the faster presentation rates throughout the first half of the list. This advantage was eliminated in the second half.

Finally, individual differences were analyzed. The mean proportion of words correctly recalled and the mean maximum number of words rehearsed correctly in sequence were calculated for each participant for the medium and slow rates. Figure 5 shows the strong positive correlations between the maximum rehearsed sequence length and the overall proportion correct for both the medium $(r=.67$, $\left.r^{2}=.45, p=.001\right)$ and slow $\left(r=.77, r^{2}=.59, p<.001\right)$ rates.
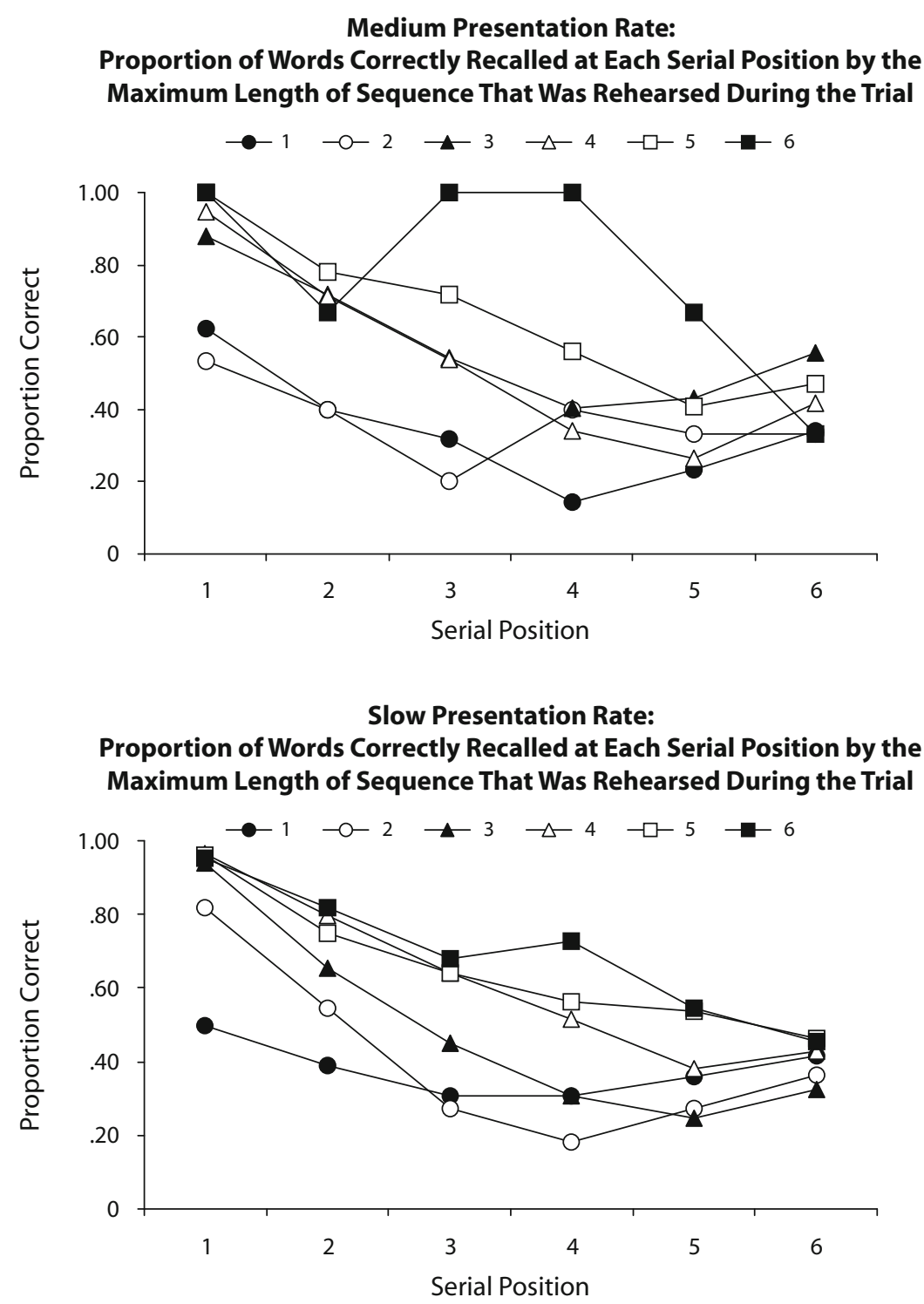

Figure 3. Data from the overt rehearsal conditions. The mean proportion of words recalled in order, plotted as a function of serial position and the maximum rehearsed sequence length during the trial. A score of 5 on this measure indicates that at any point during a trial, the longest sequence of words rehearsed in correct order, starting from the first, was five. Data in the upper panel are for the medium presentation rate, and data in the lower panel are for the slow presentation rate. 


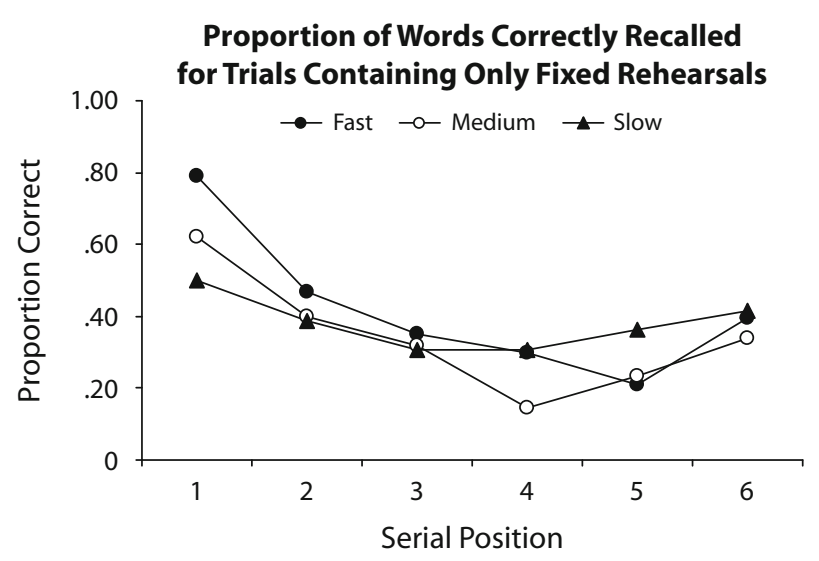

Figure 4. Data from the overt rehearsal conditions in which only the fixed rehearsal strategy was used throughout the trial. The mean proportion of words recalled in the correct order is plotted as a function of the presentation rate and serial position.

Table 1 shows these correlations for each serial position. As can be seen, these correlations were relatively high for Serial Positions 1-4 and were reduced at Serial Positions 5 and 6.

\section{DISCUSSION}

There are four main findings from the present experiment. First, considering the rehearsal data, when participants rehearse aloud during ISR, they tend to adopt either a fixed rehearsal strategy or a cumulative forward-order strategy. At fast rates (and to a lesser extent at slower rates), participants tend to rehearse only the currently presented item (fixed rehearsal strategy). However, at slower rates, when this does not take place, they tend to rehearse in cumulative forward order. That is, they rehearse the maximum possible sequence of items in forward serial order during each RS, up to RS 4. At RS 5 and RS 6, however, participants appear to refrain from rehearsal, rehearse only incomplete sequences (i.e., sequences shorter than five), or rehearse shorter sequences of later items. These patterns are consistent with the assumptions of Page and Norris (1998): Participants rehearse in a full cumulative forward order only in the early part of a list.

Table 1

Correlations Between the Proportions of Words Recalled in the Correct Order and the Maximum Sequence Length to Which the Words Were Rehearsed, for the Words Presented in Each of the Six Serial Positions, and for Both the Medium and Slow Presentation Rates

\begin{tabular}{|c|c|c|c|c|}
\hline \multirow{3}{*}{$\begin{array}{c}\text { Serial } \\
\text { Position }\end{array}$} & \multicolumn{4}{|c|}{ Presentation Rate } \\
\hline & \multicolumn{2}{|c|}{ Medium } & \multicolumn{2}{|c|}{ Slow } \\
\hline & $r$ & $r^{2}$ & $r$ & $r^{2}$ \\
\hline 1 & $.62^{* *}$ & .39 & $.81^{* * *}$ & .65 \\
\hline 2 & $.62^{* *}$ & .38 & $.53^{*}$ & .28 \\
\hline 3 & $.54^{*}$ & .30 & $.63^{* *}$ & .39 \\
\hline 4 & $.68^{* *}$ & .47 & $.68^{* *}$ & .46 \\
\hline 5 & .33 & .11 & $.48^{*}$ & .23 \\
\hline 6 & .31 & .09 & .25 & .06 \\
\hline
\end{tabular}

${ }^{*} p<.05 . \quad{ }^{* *} p<.01 . \quad{ }^{* * *} p<.001$.
Second, ISR performance was superior overall at the slow rate compared with the fast rate. However, the precise relationship between rate and recall appears complex. A slow rate might be expected to reduce recall due to the increased retention interval, but also to increase recall due to the increased opportunity to rehearse. Our data suggest that, on average, the increase in accuracy afforded by rehearsal more than offset the reduction in accuracy attributable to longer retention intervals. When one considers ISR performance across presentation rates in the absence of cumulative forward rehearsal (Figure 4), we find that recall accuracy is indeed reduced with slower presentation rates. However, many participants tended to engage in rehearsal at slower rates, and within each slower rate, ISR performance increased with increased forwardorder rehearsal (Figure 3). Furthermore, there are strong positive correlations between the rehearsal of sequences of words and their subsequent recalls, suggesting that increased rehearsal counteracts the effects of increased retention interval at slower rates.

Third, the general shape of the serial position curve in ISR was similar for all presentation rates, including the fast rate, in which there was little or no rehearsal. This suggests that the extended primacy observed in ISR need not always be a direct consequence of increased rehearsal (see also, e.g., Coltheart \& Langdon, 1998). This finding contrasts with that typically observed with free recall of longer lists (Rundus, 1971; Tan \& Ward, 2000), in which there is a close relationship between rehearsal and extended primacy effects. Rather, we believe that other factors, such as the order of recall, which was identical across all presentation rate conditions, has a large effect on the shape of the serial position curve in ISR (e.g., Tan \& Ward, 2007).

Finally, our demonstration that performance breaks down in rehearsal and recall in similar ways supports the notion that similar mechanisms underpin rehearsal and recall in ISR (Burgess \& Hitch, 1999). Rehearsal of subspan length lists (four items or fewer) is near perfect in our data, matching the nearly perfect recall performance normally found on such short lists (Miller, 1956). However, when the list length increases to five or six, rehearsal is no longer perfect. Earlier items can still be rehearsed in correct order, but rehearsal tends to break down toward the end of the list. (This may be simply an artifact of the limited time available to rehearse all items during the interstimulus interval.) We observe that the recall data suggestively mirror this pattern.

One difficulty with interpreting the positive correlations observed between overt rehearsals and subsequent recalls is that a number of alternative factors could lead to relations being seen between rehearsal and recall, even if the former is not causing the latter. A number of potential confounds include subject- or item-selection effects, or trial-by-trial fluctuations in motivation or attention. Apparent correlations between rehearsal and recall could reflect the fact that more able participants rehearse more and then recall more than do less able participants. Alternatively, participants may be more motivated on some lists than on others or find some words to be easier to rehearse 

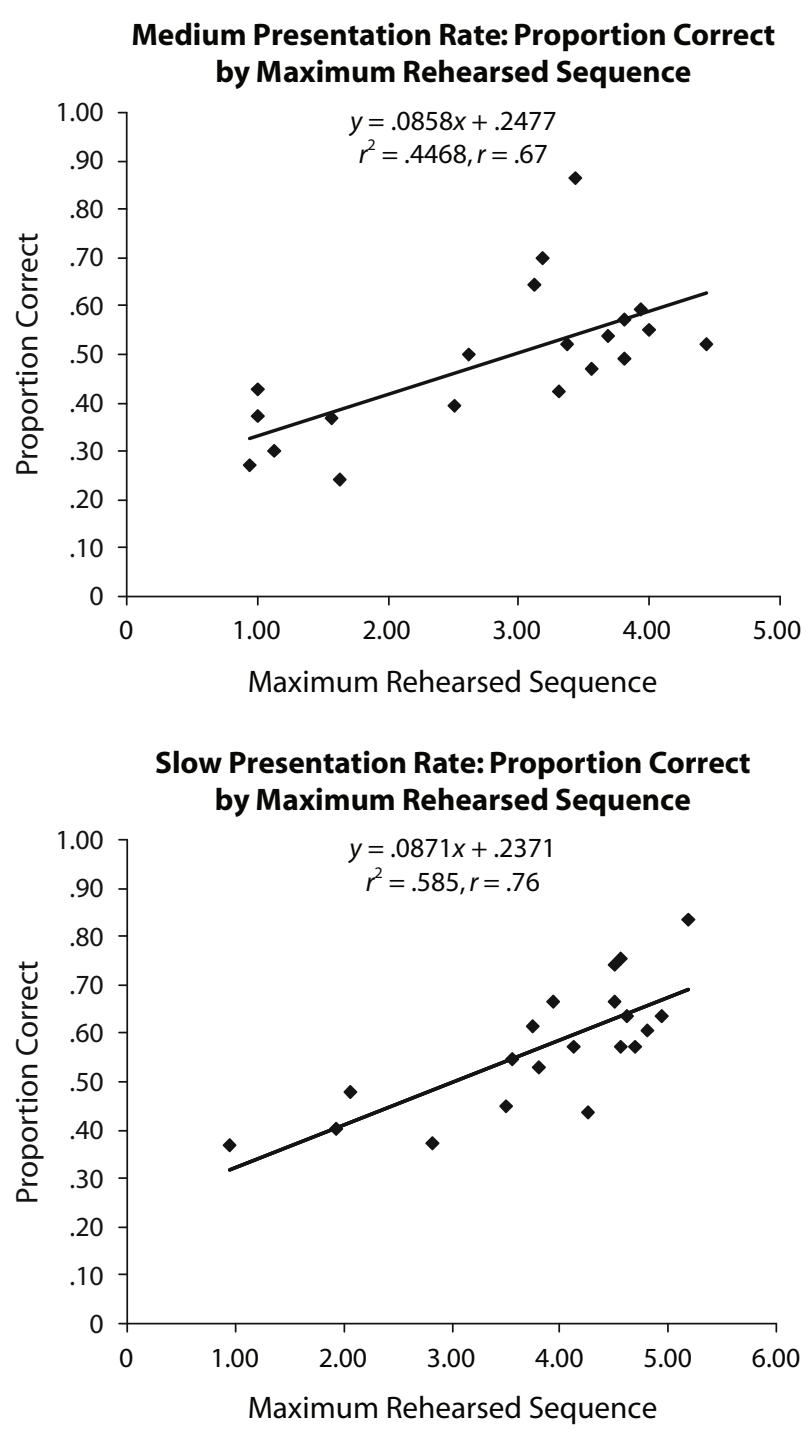

Figure 5. Data from the overt rehearsal conditions. Scatterplots showing the relationship between the mean proportion correct immediate serial recall performance and the mean length of the maximum sequence of words rehearsed in the correct order, for the medium (top) and slow (bottom) presentation rates.

and remember than others. The controlled rehearsal (Murdock \& Metcalfe, 1978) and yoked rehearsal procedures (Tan \& Ward, 2000) have alleviated some similar concerns in free recall. In these procedures, recall is similar when participants can freely select which words to rehearse, and when they are presented with schedules of repetitions over which they have no control.

We have provided the first detailed descriptions concerning the words rehearsed during ISR. This information can be used to help constrain and test different computational ISR models. More specifically, these data tend to support the assumptions regarding participants' rehearsal strategies in the primacy model (Page \& Norris, 1998), and indicate similarities between rehearsal and recall (Burgess $\&$ Hitch, 1999). Our results mirror those found in recent examinations of similarities and differences between rehearsal and recall examinations of free recall (Laming, 2006; Tan \& Ward, 2000). It is often assumed that ISR can be differentiated from free recall because of the requirement for forward serial-order recall in the former but not in the latter. However, over the last decade or so, evidence has been emerging supporting a tendency for forwardorder output even in free recall (Beaman \& Morton, 2000; Bhatarah, Ward, \& Tan, 2008; Howard \& Kahana, 1999; Klein, Addis, \& Kahana, 2005; Ward, Woodward, Stevens, \& Stinson, 2003), and we believe that there are grounds for suggesting that the processes underpinning the recall and rehearsals in free recall and ISR may be more similar than has been assumed.

\section{AUTHOR NOTE}

The authors were supported by ESRC Research Grant R000239674. Correspondence should be addressed to L. Tan, Department of Psychology, City University, Northampton Square, London EC1V 0HB, England (e-mail: lydia.tan.1@city.ac.uk) or G. Ward, Department of Psychology, University of Essex, Wivenhoe Park, Colchester CO4 3SQ, England (e-mail: gdward@essex.ac.uk).

\section{REFERENCES}

BAdDEley, A. [D.] (1986). Working memory. Oxford: Clarendon Press. BADDEley, A. [D.], \& LeWIS, V. [J.] (1984). When does rapid presentation enhance digit span? Bulletin of the Psychonomic Society, 22, 403-405.

Baddeley, A. D., Lewis, V. [J.], \& Vallar, G. (1984). Exploring the articulatory loop. Quarterly Journal of Experimental Psychology, 36A, 233-252.

Baddeley, A. D., Thomson, N., \& Buchanan, M. (1975). Word length and the structure of short-term memory. Journal of Verbal Learning \& Verbal Behavior, 14, 575-589.

Beaman, C. P., \& Morton, J. (2000). The separate but related origins of the recency effect and the modality effect in free recall. Cognition, 77, B59-B65.

Bhatarah, P., Ward, G., \& TAn, L. (2008). Examining the relationship between free recall and immediate serial recall: The serial nature of recall and the effect of test expectancy. Memory \& Cognition, 36, 20-34.

Burgess, N., \& Hitch, G. J. (1999). Memory for serial order: A network model of the phonological loop and its timing. Psychological Review, 106, 551-581.

ColtheART, V., \& LANGDON, R. (1998). Recall of short word lists presented visually at fast rates: Effects of phonological similarity and word length. Memory \& Cognition, 26, 330-342.

Conrad, R., \& Hille, B. A. (1958). The decay theory of immediate memory and paced recall. Canadian Journal of Psychology, 12, 1-6.

Friendly, M., Franklin, P. E., Hoffman, D., \& Rubin, D. C. (1982). The Toronto Word Pool: Norms for imagery, concreteness, orthographic variables, and grammatical usage for 1,080 words. Behavior Research Methods \& Instrumentation, 14, 375-399.

Henson, R. N. A. (1998). Short-term memory for serial order: The startend model. Cognitive Psychology, 36, 73-137.

HowARD, M. W., \& Kahana, M. J. (1999). Contextual variability and serial position effects in free recall. Journal of Experimental Psychology: Learning, Memory, \& Cognition, 25, 923-941.

Klein, K. A., Addis, K. M., \& Kahana, M. J. (2005). A comparative analysis of serial and free recall. Memory \& Cognition, 33, 833-839.

KuČERA, H., \& Francis, W. N. (1967). Computational analysis of present-day American English. Providence, RI: Brown University Press.

LAMING, D. (2006). Predicting free recalls. Journal of Experimental Psychology: Learning, Memory, \& Cognition, 32, 1146-1163.

Mackworth, J. F. (1962). Presentation rate and immediate memory. Canadian Journal of Psychology, 16, 42-47. 
Miller, G. A. (1956). The magic number seven, plus or minus two: Some limits on our capacity for processing information. Psychological Review, 63, 81-97.

Murdock, B. B., \& Metcalfe, J. (1978). Controlled rehearsal in single-trial free recall. Journal of Verbal Learning \& Verbal Behavior, 17, 309-324.

Page, M. P. A., \& Norris, D. (1998). The primacy model: A new mode of immediate serial recall. Psychological Review, 105, 761-781.

PAlmer, S. E., \& Ornstein, P. A. (1971). Role of rehearsal strategy in serial probed recall. Journal of Experimental Psychology, 88, 60-66.

Rundus, D. (1971). Analysis of rehearsal processes in free recall. Journal of Experimental Psychology, 89, 63-77.
TAN, L., \& WARD, G. (2000). A recency-based account of the primacy effect in free recall. Journal of Experimental Psychology: Learning, Memory, \& Cognition, 26, 1589-1625.

TAN, L., \& WARD, G. (2007). Output order in immediate serial recall. Memory \& Cognition, 35, 1093-1106.

Ward, G., Woodward, G., Stevens, A., \& Stinson, C. (2003). Using overt rehearsals to explain word frequency effects in free recall. Journal of Experimental Psychology: Learning, Memory, \& Cognition, 29, 186-210.

(Manuscript received November 27, 2006; revision accepted for publication November 5, 2007.) 